\title{
Sleep dysfunctions in schizophrenia: A practical review
}

\author{
Flavie Waters ${ }^{1,2}$, Dara S. Manoach ${ }^{3,4,5}$ \\ ${ }^{1}$ Centre for Clinical Research in Neuropsychiatry, Graylands Hospital, North Metro Area Mental Health, Perth, Australia \\ ${ }^{2}$ School of Psychiatry and Clinical Neurosciences, The University of Western Australia, Perth, Australia \\ ${ }^{3}$ Department of Psychiatry, Massachusetts General Hospital, Charlestown, USA \\ ${ }^{4}$ Athinoula A. Martinos Center for Biomedical Imaging, Charlestown, USA \\ ${ }^{5}$ Harvard Medical School, Boston, USA \\ Email: flavie.waters@health.wa.gov.au
}

Received 20 September 2012; revised 18 October 2012; accepted 26 October 2012

\section{ABSTRACT}

Objective: Sleep dysfunctions are common in schizophrenia, yet few evidence-based guidelines exist for the detection, diagnosis and management of sleep disturbances in this disorder. This critical review paper sought to increase awareness amongst mental health clinicians of sleep problems in schizophrenia, promote better identification of sleep problems, and help clinicians make more informed decisions about integrated treatment plans. Specifically, we examined the following key questions: 1) Which sleep problems occur in schizophrenia? 2) What evidence exists regarding the impact of poor sleep? 3) What are the underlying mechanisms of sleep problems in schizophrenia? 4) How can this information be used by clinicians to design a more complete treatment plan? Data Sources and study selection: We conducted a non-systematic review of studies that have shaped our current understanding of sleep in schizophrenia $(n=$ 65). Data sources included PubMed, Medline, and cross-referencing. Results: Sleep disorders are pervasive and broad-ranging in people with schizophrenia, and are associated with substantial burden. An integrated model is proposed whereby a combination of highly interactive factors comprising genetic and neurobiological vulnerabilities, and behavioural and environmental factors, interact to cause sleep abnormalities. However, prospective and rigorous studies of sleep in schizophrenia are lacking. Conclusion: Patients often do not receive optimal care because sleep problems are rarely diagnosed, and treatments plans are incompletely formulated. A better understanding of sleep problems in schizophrenia will lead to increased treatment options, and a more positive clinical outcome.

Keywords: Circadian; Sleep Wake; Medication; PSG; Spindles; Cognition; OSA

\section{INTRODUCTION}

Schizophrenia (SZ) is a persistent and debilitating psychotic illness that causes devastation in the lives of sufferers and their families. The personal costs to the individual in terms of suffering and disability are also enormous, with individuals experiencing isolation, financial hardship, unemployment and periods of hospitalisation. Symptoms include psychotic symptoms ("voices", bizarre beliefs), disorganised thoughts and negative symptoms. Sleep problems are also a prominent clinical feature of the disorder, first described more than century ago by Kraepelin and Bleuler. The first empirical studies of sleep in SZ were led by Feinberg's polysomnography studies in the 1960s. Since then, studies have showed that sleep dysfunctions occur regardless of age, phase of the illness, or medication regime. Several high quality reviews on the topic exist [1-3], but these have typically focused on specific domains of sleep, resulting in an incomplete understanding of sleep problems in SZ. The paucity of reviews on the clinical significance of abnormal sleep and available treatment options in SZ has perhaps contributed to lack of translation from the bench to the clinic. Yet, the importance of this topic lies in the facts that sleep problems are remediable, and that sleep treatments have the potential to lead to significant improvements to clinical outcomes.

Given the increasing interest in the nature and correlates of sleep disorders, an up-to-date review of sleep abnormalities in SZ is urgently needed. Many more papers have been published on the topic than this review can include. This paper thus presents a non-systematic review of studies which have shaped current knowledge about sleep and SZ and which address the following questions: 1) Which sleep problems exist in schizophrenia? 2) What is the impact of poor sleep? 3) What are the underlying mechanisms of sleep problems in SZ? 4) How can this information be used by clinicians to design a more integrated treatment plan? Overall, we highlight sleep issues as an important dimension in $\mathrm{SZ}$ with direct 
relevance for mental health clinicians. A better understanding of sleep problems in SZ patients will lead a better identification of sleep problems, and more informed decisions about integrated and complete treatment plans. It will also help clinicians think more about the interacttion in the fields of sleep and psychiatry.

\section{WHICH SLEEP PROBLEMS OCCUR IN SZ?}

\subsection{Insomnia and Circadian Rhythm Disorders}

Clinical observations, subjective reports, and questionnaire studies have been remarkably consistent in showing difficulties falling sleep ("increased sleep latency"), sleep interruptions, and longer need for sleep [4-6] in SZ. Indeed, studies suggest that approximately 50\% - 70\% of individuals with SZ experience insomnia [6]. Objective assessments of sleep-wake patterns also corroborate these reports. Actigraphy monitoring, which allows $24 \mathrm{hr}$ online recording of wrist movements from which sleep is inferred, provides evidence that increased sleep latency and difficulties in maintaining sleep are a characteristic feature of the disorder in medicated and unmedicated individuals e.g. [5,7]. The evidence also suggests that reduced sleep duration is more commonly reported in neuroleptic-naive or drug-free individuals, while a longer need for sleep is reported in medicated samples $[4,8]$, consistent with drug studies demonstrating the sedative properties of antipsychotic medication. Investigations of circadian rhythm chronobiologic parameters in SZ also point to disruptions in the internal biological clock of patients, showing dysregulation of the suprachiasmatic nucleus (SCN) and abnormal levels of melatonin [9-12].

Insomnia has been noted as one of the earliest clinical symptom that heralds the onset of psychosis, and possibly relapse [13], yet these sleep symptoms are not pathognomonic for the disorder. Insomnia is a key feature of other psychiatric disorders such as depression and bipolar disorder. In fact, studies suggest that the rates of insomnia are reported to be even higher in affective disorder (69\% to $99 \%)$ [14] than in SZ.

\subsection{Sleep Architecture}

Sleep architecture abnormalities are frequently reported in SZ. The most widely reported is a decrease in the amount of deep, slow wave sleep (SWS) [1] in antipsychotic-naïve, unmedicated, medicated and remitted patients. Rapid-eye movement (REM) sleep abnormalities have also been reported, including reduced latency to the onset of the first REM period [15-17]. However, negative findings also exist and neither SWS nor REM sleep abnormalities are consistently observed; in addition, neither survive meta-analyses of SZ patients and healthy or psychiatric controls [5,7].
Sleep architecture abnormalities lead to abnormal control of the sleep-wake systems, and thus lead to a range of sleep phenomena including sleep fragmentation, narcolepsy, sleep paralysis and other nocturnal sleep conditions. Interestingly, the overlap in symptoms between narcolepsy and schizophrenia has been noted. These include vivid hallucinations due to the intrusion of REM into wakefulness, bizarre behaviours and significant disability, similar to the clinical picture observed in the disorganized type of SZ. However, there are several ways to differentiate narcolepsy from schizophrenia: 1) narcolepsy symptoms are resistant to antipsychotic medications; 2) tests are available in sleep clinics to test for narcolepsy, including Mean Sleep Latency Testing (MSLT), human leukocyte antigen (HLA) typing.

\subsection{Spectral Characteristics of Sleep}

Only a few studies have gone beyond sleep architecture to examine the spectral characteristics of the sleep electroencephalogram (EEG) in SZ. There have been reports of reduced slow wave activity [18] during SWS in SZ. There is also accumulating evidence of reduced sleep spindles in SZ. Some early studies found no differences between patients and controls, e.g. [19] although such studies used small samples $(<11)$ of unmedicated patients and identified NREM spindles with visual detection at a single EEG channel. In contrast, recent studies that used automated spindle detection algorithms, a larger number of EEG channels, and larger samples comprised of medicated SZ patients report a dramatic reduction of sleep spindles in SZ [20,21], providing evidence of a spindle deficit in SZ that is unlikely to be due to antipsychotic medication. The clinical and functional significance of this deficit needs to be a focus of future research, although a recent hypothesis links sleep spindles to the thalamic reticular nucleus (TRN) circuitry dysfunction in SZ, e.g. [22]. Given the link between TRN circuits and attention and sensory gating, one interesting area of research might involve an examination of sleep spindles in SZ and the regulation of attention.

\subsection{Obstructive Sleep Apnoea}

Obstructive sleep apnoea (OSA) refers to a sleep-related breathing disorder characterised by abnormal respiratory events including abnormal pauses of breathing, and a cessation of airflow. Several studies of OSA in SZ exist $[23,24]$, which point to significantly elevated rates of OSA in these populations. Estimates range between 25\% reported by Waters et al. [25] in an audit of SZ inpatients using self-reports, and 48\% reported by Ancoli-Israel et al. (1999) [24] using a portable sleep recording device in 52 older patients with schizophrenia (mean age of 59.6 years). In an examination of the Veterans Health Admini- 
stration database, Sharafkhaneh et al. [26] found that a diagnosis of psychosis (including schizophrenia and schizo-affective disorders) increased the odds ratio of having sleep apnoea by 1.35 , which was independent of substance abuse, age or sex.

Indirect evidence also points to an increased risk of OSA in SZ. Obesity is the highest predictor for OSA, and it is also a common consequence of treatment with antipsychotic drugs. It is therefore not surprising that studies show that OSA is linked to higher body mass index in SZ [27], and to antipsychotic-induced obesity [25,28]. Other risk factors for OSA include alcohol consumption, use of hypnotics and smoking, which are common in SZ, and thus further increase the risk for OSA.

Overall, OSA in SZ is often overlooked and left untreated. Its associated symptoms (awakenings, sleepiness and disruptions to the timing and quality of sleep) are often attributed to other sleep disorders, lifestyle factors, or side effects from medication, and OSA continues to be poorly recognized by clinicians.

\subsection{Movement-Related Sleep Disorders}

Restless Legs Syndrome (RLS) and Periodic Limb Movements Syndrome (PLMS) refer to separate conditions characterised by unpleasant leg sensations and repeated jerking of the legs during sleep. Terms describing RLS sensations, such as ants crawling or worms moving under the skin, are similar to somatic hallucination descriptions, though the occurrence of these symptoms in the evening/night, and in the legs, is necessary for a diagnosis of RLS. A diagnosis of RLS and PLMS is also typically based on a PSG. There are very few studies of movement-related sleep disorders in SZ, and estimates of RLS and PLMS range between $13 \%$ to $21 \%$ [29]. The exact neurobiological mechanisms are not understood, although one hypothesis proposes that dopamine deficiency is a likely cause [30]. This has given rise to the "depletion" theory which posits that antipsychotics in SZ may precipitate such problems through the blocking of dopamine (DA) [27]. In support, a number of case studies have linked sleep-related movement disorders to atypical antipsychotic medication, where symptoms reductions were reported after switching to a different antipsychotic drug [31].

\subsection{Effects of Medication on Sleep}

Sleep dysfunctions are a clearly important clinical dimension of SZ, yet pharmacological treatments for SZ also appear to have prominent effects on sleep [2]. For example, typical and atypical antipsychotics have also been demonstrated to show beneficial effects on sleep, by increasing sleep efficiency and total sleep time, and reducing sleep onset latency (SOL) [32-34]. While these findings promote the positive effects of antipsychotics on sleep, abnormalities of sleep timing and architecture still persist when compared non-psychiatric controls. For example, neurobiological circadian parameters and sleep spindle abnormalities remain uncorrected $[9,12,35]$. In addition, a recent study examined the relationship between the number (and dosage) of antipsychotic medications and sleep in a psychiatric inpatient population including SZ patients. The results showed that increasing antipsychotics dosages were linked to better sleep, although antipsychotic medications only accounted for a relatively small amount of the variance in sleep (6\%) [36]. Furthermore, sleep complaints were pervasive in $70 \%$ of this medicated clinical sample. These findings suggest that the use of the sedative properties of antipsychotic medication has limited efficacy as a treatment option for sleep dysfunctions, and is not an appropriate substitute for sleep interventions.

Other drugs have opposite effects on sleep. Selective serotonin-reuptake inhibitors (SSRI) and the serotoninnorepinephrine-reuptake inhibitors (SNRI), for example, have alerting effects, and are linked to fragmented sleep, increased SOL and daytime somnolence [33]. However, in an examination of the effect of polypharmacy on sleep in psychiatric inpatients, Waters et al. [36] showed that SSRI/SNRI, when taken concurrently with antipsychotic medications, failed to show disruptive effect on sleep pattern or daytime functions. A gradual tolerance of sleep effects of SSRI in chronic treatment remains a possibility was provided as one explanation for this finding [37].

\section{WHAT IS THE IMPACT OF POOR SLEEP IN SZ?}

Sleep disorders in the general population are linked to health risks and functional impairments [38]. Surprisingly, the functional impact of sleep disorders in SZ remains little investigated, although estimates suggest that poor sleep may account for $24 \%$ of the variance in quality of life in SZ [39]. Exceptions include studies linking poor sleep in SZ, as assessed using questionnaires, to reduced functional and psychosocial outcomes including decreased coping and increased risk for suicide [39,40].

Cross-sectional studies of SZ have also demonstrated a link between sleep architecture and psychopathology [41-43]. The link between positive symptoms and REM sleep has been of interest for decades due to similarities between dreams and psychosis [44]. An early hypothesis suggested that SZ was determined by a dysregulation of REM sleep [15,23]. Overnight sleep studies have confirmed that REM abnormalities are linked to positive symptoms, although the functional mechanisms remain unclear.

Recent interest in spectral characteristics have linked spindle deficits to greater severity of positive symptoms, 
which was absent in a non-SZ psychiatric control group treated with antipsychotic drugs [45]. A recent study [46] however found no significant correlation between sigma power which corresponds to spindles, and severity of positive and negative symptoms in 27 untreated psychotic patients (15 with SZ). Clearly, the relationship between spindle deficit and psychotic symptoms requires further explication.

Anecdotally, patients report that poor sleep is linked to a next-day increase in paranoia and intensity of auditory hallucinations. This is corroborated by behavioural or longitudinal studies which show significant correlations between positive symptoms and reduced sleep duration, sleep latency or efficiency $[47,48]$. Negative symptoms, by contrast, have tended to be linked to SWS [49], although some studies have also found shortened REM latency, e.g. [49]. Clearly, some relationship exists between sleep parameters and clinical characteristics, although comprehensive functional explanations are still lacking.

The relationship between sleep and cognition is an important area of research given that cognitive impairments in SZ are predictive of poor functional outcome. The timing, and architecture, of sleep have been linked to performance on broad neuropsychological tests in SZ. Deficits on test of attention and response times are the most commonly reported findings [10], and are in agreement with the literature in healthy people showing that sleep deficits cause reliable reductions in speed of processing and attention. Increasing evidence also links impairments in slow waves and sleep spindles with attention [46], but not with general cognitive ability. These findings are consistent with the hypothesis of thalamic reticular nucleus (TRN) circuitry dysfunction that predicts deficits in attention regulation processes [22].

There has been recent interest in memory consolidation processes, in view of evidence that sleep is important for learning and memory. Initial memory encoding is followed by a prolonged period of consolidation, integration, and reorganization that continues over days, months, or even years. Much of this evolution of memories is thought to be mediated by sleep. Evidence shows that memories may be replayed, consolidated, and even improved, during sleep, so that one proposed function of sleep is that it may be involved in the laying-down of memories from a short-term state to a more permanent form. In SZ, there is evidence of impaired memory consolidation across multiple domains [50]. Emerging evidence suggests that sleep abnormalities may contribute to these deficits. For example, SZ patients have marked impairments of sleep-dependent motor procedural memory consolidation [51] and the reduction in spindle activity predicts reduces sleep-dependent memory consolida- tion. Slow wave activity and sleep spindles in healthy people have been correlated with overnight improvement of motor procedural learning and accumulating evidence suggests that sleep spindles mediate sleep-dependent consolidation of both procedural and declarative memory. Overall, evidence links sleep spindles to memory dysfunctions in SZ, although the extent to which abnormal sleep contributes to other aspects of cognitive dysfunction in SZ remains unclear.

\section{WHAT ARE THE MECHANISMS UNDERLYING SLEEP PROBLEMS IN SZ?}

There are several hypothesised interactive pathways linking sleep problems with SZ characteristics (Figure 1).

\section{Neurobiological, Genetic and Environmental Factors}

Neurobiological abnormalities underlie some of the circadian sleep-wake problems in SZ. The suprachiasmatic nucleus (SCN) is the master circadian pacemaker, whose role consists in orchestrating levels of hormones like melatonin, cortisol and prolactin, and core body temperatures according to a fixed cycle that is synchronised by environmental cues. As noted above, investigations of circadian rhythm chronobiologic parameters in SZ have identified dysregulation of the SCN, including abnormal 24-hr rhythms (phase advance) of melatonin and serotonin, body temperature, cortisol, and prolactin e.g. [9,11,12,35,52]. In addition to the role of serotonin dysfunctions, deficits of the inhibitory neurotransmitter gamma amino butyric acid (GABA) may play a role in SZ sleep dysfunctions. GABAergic alterations are well documented in SZ, and some researchers have pointed to animal and human studies linking GABA to sleep architecture and homeostatis, suggesting a potential role of GABA receptors in SZ sleep abnormalities.

Several circadian timekeeping genes such as PERIOD and CLOCK have been identified in the human SCN, and may also be involved in the pathophysiology of SZ e.g. [53]. Associations between CLOCK genes and SZ may possibly occur through dopaminergic transmission to the SCN that is linked to CLOCK gene-activity [54]. One study linked a haplotype analysis of CLOCK gene with female patients with SZ, although the statistical significance did not hold after correction for multiple comparisons [55]. An association between PERIOD genes and SZ has also been identified, with weak-tomoderate effects [53].

An examination of the heritability of sleep problems in SZ also points to familial factors. The PSG data of SZ and their first degree relatives showed abnormal SWS 
Endogenous factors

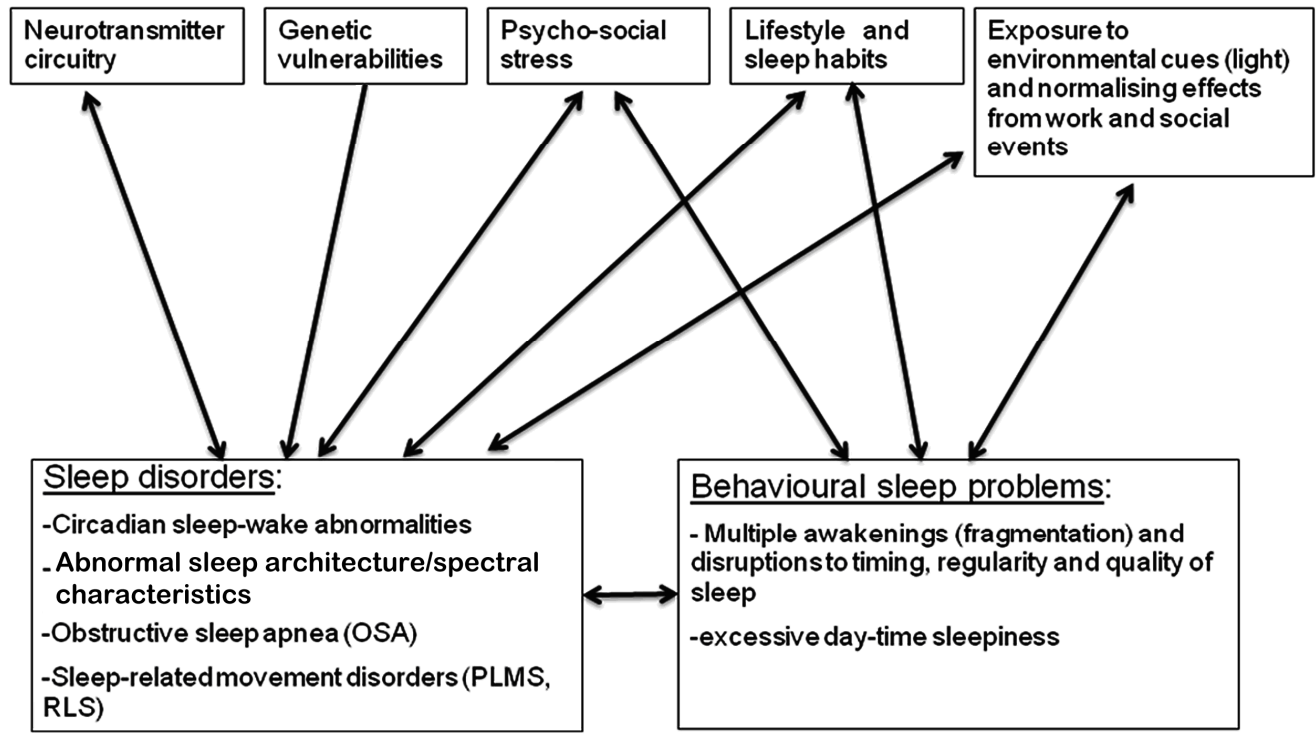

Figure1. Mechanisms of poor sleep in schizophrenia.

\section{Exogenous factors}

duration that was different from healthy controls [56]. These findings confirmed results of an early study of sleep abnormalities in relatives of SZ, that showed increased awaking after sleep onset $(\mathrm{p}=0.06)$, reductions in SWS, decreased REM\%, and decreased REM counts [57]. Thus, genetic factors may provide a direct vulnerability for experiencing sleep problems, via chronobiological and neurobiological changes leading to circadian sleep-wake disorders. Indirect genetic effects may also predict poor sleep via a vulnerability to experiencing stress.

While there are strong genetic factors associated with OSA in the general population, perhaps linked to the angiotensin converting enzyme (ACE) gene polymerphism , SZ studies of OSA genes have not yet been reported. The relative contribution of medication effects and genetic factors to the risk of OSA remains unclear at the point.

Environmental factors also make a significant contribution towards poor sleep in SZ. Normally, the onset of night and day, and other socially-determined rhythms ("zeitgebers"), are important contributors by imposing regular timing on circadian sleep-wake system. The disability in SZ, linked to a lack of regular employment, irregular timing of meal times and social activities, abnormal exposure to light and other social rhythms, may perpetuate or accentuate sleep problems by affecting timekeeping mechanisms. Symptoms of depression and anxiety also contribute to a lack of entrainment and disruption of biological rhythms.

Behavioural contributors include activities that are inconsistent with the maintenance of healthy sleep. Excessive napping, irregular sleep timing, excessive caffeine consumption, poor diet, obesity, stress, alcohol, smoking and drug use, all have a measurable impact on sleepwake patterns occurring at abnormal times, although they are unlikely to account for the entire range and severity of sleep disorders in SZ. Lifestyle factors also contribute to fat and weight, and impacts of respiratory changes and sleep disordered breathing.

A range of primary and secondary sleep issues may be observed. Primary sleep disorders result from an endogenous abnormality in timing, biological or physiological mechanisms, while secondary sleep disorders are often caused by an underlying physical or mental condition, or a primary sleep disorder. The differentiation between primary and second sleep disorders however is not always easy, especially in psychiatric disorders. For example, OSA can be considered secondary to obesity and metabolic factors. Insomnia is a primary sleep disorder, but may develop as a consequence of multiple awakenings and lack of restorative sleep due to conditions such as depression, anxiety, and sleep disorders such as OSA and RLS. Both primary sleep disorders, and secondary sleep problems, can cause behavioural, clinical, cognitive and functional deficits, although their relative contribution is not well understood. Primary sleep disorders might have specific effects on functions. One example is the association between REM latency and positive symptoms, via dopaminergic and GABAergic effects. Another example includes the link between REM on memory, via the selective effects of REM on synaptic plasticity in the hippocampus which allows a redistribution of memory representations to neocortical networks. Finally, OSA may cause executive dysfunctions due to the effects of hypoxia on frontal networks. By contrast, 
behavioural sleep problems may have a broader and more diffuse impact by causing destabilizations on central functions that impact on the efficiency of the brain systems, leading to broad changes in psychopathology, cognition and thus functioning.

Together, a combination of highly interactive factors links sleep problems to a host of physical and mental health problems.

\section{HOW CAN THIS INFORMATION BE USED TO HELP PEOPLE MAY WITH THE DISORDER?}

Many of the sleep dysfunctions described above, and the factors contributing to these sleep problems, are not specific for schizophrenia, and therefore have limited value for diagnostic purposes. However, sleep problems have a significant clinical impact at the individual level and must be addressed clinically. Beyond its consequences on quality of life and functioning, and contribution to relapse, the failure to resolve sleep issues can have direct consequences on health. Existing sleep disorders may impact on the degree to which individuals benefit from cognitive rehabilitation and other medical therapies though this type of information is not yet available in SZ. In addition, sleep problems may cause further complications if they remain undiagnosed, as they may hasten progression of clinical dysfunctions, or increase the risk of CVD, stroke and mortality, and further degrade quality of life.

The benefits of knowledge about sleep difficulties include better decision-making regarding treatment. The first step in identifying sleep problems in SZ is to routinely screen for sleep problems. This might involve asking the patient to describe the nature of their sleep difficulties. Questionnaires are highly effective for screening for sleep problems, and are routinely used in clinical settings. Several questionnaires exist that assess sleep quality [58], risk of sleep apnoea [59], and impact of sleep dysfunctions on daytime functions [60,61].

The second step is to modify patients' rehabilitation plan. External factors (such as irregular sleep timing, caffeine, alcohol, drugs, and stress) can contribute to the development of secondary sleep disorders. These are modifiable risk factors that can be addressed directly without further consultation with sleep specialists, and with relatively simple sleep medicine protocols that may be incorporated in the patient rehabilitation plan. Examples include changing lifestyle, sleep hygiene and habits. Normalising sleep-wake patterns can be achieved by aligning sleep schedules with personal preferences and social/occupational requirement. These may at least reduce the principal impact of sleep dysfunctions.

Primary sleep disorders require different types of treatment and need referrals to sleep clinics. Treating the symptoms of primary sleep disorders will often improve symptoms of secondary disorders. Several types of treatments are available for sleep-wake disorders. Decisions regarding suitability must be made on a case-bycase basis, in discussion with a sleep clinician, and in consideration of concurrently-taken psychotropic medications. Interventions can include pharmacotherapy, chronotherapy or behavioural techniques. These are commonly recommended, and generally effective, in the general community, but their efficacy have rarely been tested in SZ patients. One exception is melatonin, which has been shown to improve in sleep efficiency in patients with bipolar disorder, and in patients with SZ whose sleep quality was low [12], although an interaction with antipsychotic agents has been noted [12].

OSA is highly remediable, and is a good treatment target for SZ. Treatments for OSA are relatively simple and cost-effective, and can produce dramatic improvements in functioning and quality of life. The first line of treatment for OSA is usually continuous positive airway pressure (CPAP). There are few published studies of CPAP in SZ, although these show measurable improvements in psychotic and depressive symptoms, as well as improvements in psychiatric functions [62,63]. Poor compliance is a significant issue for people with SZ, given that treatment involves lifelong use of nocturnal CPAP lasting 6 hrs or more per night. However, adherence is a problematic issue in all age groups and populations. In high-functioning people with SZ, such treatment would be highly feasible and might help achieve better daytime alertness and daily functioning. Resolving OSA in low-functioning chronic patients, however, who are at greater risk of metabolic syndrome, is a significant problem and is a grand challenge for clinicians in years to come. Alternative treatments, including lifestyle and dietary change with a focus on reducing weight, should be pursued. Clinicians should ask patients regularly about snoring, and breathing pauses during sleep, and make appropriate referrals to sleep clinicians. Particularly, the combination of atypicals medications and obesity should alert clinicians to the need to closely monitor patients for risk of OSAHS.

\section{SUMMARY AND CONCLUSION}

The review shows that sleep problems are intrinsically linked to SZ, yet the nature and consequences of sleep dysfunction in this population remain poorly understood and under-researched. Sleep problems occur regardless the clinical phase and medication regime. Although variations have been noted, the most consistent findings include insomnia, and abnormalities in sleep architecture and spindles. OSA, and movement sleep disorders, also appear to be common. Preliminary evidence links some of these sleep disorders, at least partly, to the effects of 
antipsychotic medication. Overall, evidence suggests that SZ is linked to increased rates of primary sleep disorders, although rigorous studies are urgently needed in representative samples.

The review also shows that inadequate sleep is associated with increased psychopathology, cognitive deficits and marked decreases in functioning. This is consistent with studies in the general population documenting the severe health consequences of sleep problems. A combination of highly interactive factors in SZ links sleep problems to such disability. Negative outcomes are produced by a dynamic and fluctuating combination of sleep dysfunctions arising from a mix of primary and secondary sleep disorders. As such, there may not be one single "sleep marker" for SZ, and its complex clinical picture perhaps reflects the direct and indirect effects of environmental and genetic factors.

\section{REFERENCES}

[1] Keshavan, M.S., Reynolds, C.F. and Kupfer, D.J. (1990) Electroencephalographic sleep in schizophrenia: A critical review. Comprehensive Psychiatry, 31, 34-47. doi:10.1016/0010-440X(90)90052-T

[2] Monti, J. and Monti, D. (2005) Sleep disturbance in schizophrenia. International Review of Psychiatry, 17, 247-253. doi:10.1080/09540260500104516

[3] Manoach, D.S. and Stickgold, R. (2009) Does abnormal sleep impair memory consolidation in schizophrenia? Frontiers in Human Neuroscience, 3, 1-8. doi:10.3389/neuro.09.021.2009

[4] Poulin, J., Chouinard, S., Pampoulova, T., et al. (2010) Sleep habits in middle-aged, non-hospitalized men and women with schizophrenia: A comparison with healthy controls. Psychiatry Research, 179, 274-278. doi:10.1016/j.psychres.2009.08.009

[5] Chouinard, S., Poulin, J., Stip, E., et al. (2004) Sleep in untreated patients with schizophrenia: A meta-analysis. Schizophrenia Bulletin, 30, 957-967. doi:10.1093/oxfordjournals.schbul.a007145

[6] Benson, K. (2006) Sleep in schizophrenia: Impairments, correlates, and treatment. Psychiatric Clinics of North America, 29, 1033-1045. doi:10.1016/j.psc.2006.08.002

[7] Benca, R.M., Obermeyer, W.H., Thisted, R.A., et al. (1992) Sleep and psychiatric disorders: A meta-analysis. Archives of General Psychiatry, 49, 69-70, 651-668. doi:10.1001/archpsyc.1992.01820080059010

[8] Lieberman, J.A., Stroup, T.S., McEvoy, J.P., et al. (2005) Effectiveness of antipsychotic drugs in patients with chronic schizophrenia. The New England Journal of Medicine, 353, 1209-1223. doi:10.1056/NEJMoa051688

[9] Van Cauter, E., Linkowski, P., Kerkhofs, M., et al. (1991) Circadian and sleep-related endocrine rhythms in schizophrenia. Archives of General Psychiatry, 48, 348-356. doi:10.1001/archpsyc.1991.01810280064009

[10] Bromundt, V., Koster, M., Georgiev-Kill, A., et al. (2011)
Sleep-wake cycles and cognitive functioning in schizophrenia. The British Journal of Psychiatry, 198, 269-276.

[11] Rao, M.L., Gross, G., Strebel, B., et al. (1994) Circadian rhythm of tryptophan, serotonin, melatonin, and pituitary hormones in schizophrenia. Biological Psychiatry, 35, 151-163. doi:10.1016/0006-3223(94)91147-9

[12] Anderson, G. and Maes, M. (2012) Melatonin: An overlooked factor in schizophrenia and in the inhibition of anti-psychotic side effects. Metabolic Brain Disease, 27, 113-119. doi:10.1007/s11011-012-9307-9

[13] Chemerinski, E., Ho, B.C., Flaum, M., et al. (2002) Insomnia as a predictor for symptom worsening following antipsychotic withdrawal in schizophrenia. Comprehensive Psychiatry, 43, 393-396. doi:10.1053/comp.2002.34627

[14] Harvey, A.G. (2008) Sleep and circadian rhythms in bipolar disorder: Seeking synchrony, harmony, and regulation. America Journal of Psychiatry, 165, 820-829. doi:10.1176/appi.ajp.2008.08010098

[15] Feinberg, I., Koresko, R.L. and Gottlieb, F. (1965) Further observations on electrophysiological sleep patterns in schizophrenia. Comprehensive Psychiatry, 58, 21-24.

[16] Yang, C. and Winkelma, J.W. (2006) Clinical significance of sleep EEG abnormalities in chronic schizophrenia. Schizophrenia Research, 82, 251-260. doi:10.1016/j.schres.2005.10.021

[17] Talih, F.R. (2011) Narcolepsy presenting as schizophrenia: A literature review and two case reports. Innovations in Clinical Neuroscience, 8, 30-34.

[18] Goder, R., Aldenhoff, J.B., Boigs, M., et al. (2006) Delta power in sleep in relation to neuropsychological performance in healthy subjects and schizophrenia patients. The Journal of Neuropsychiatry and Clinical Neurosciences, 18, 529-535. doi:10.1176/appi.neuropsych.18.4.529

[19] Poulin, J., Daoust, A.M., Forest G, et al. (2003) Sleep architecture and its clinical correlates in first episode and neuroleptic-naive patients with schizophrenia. Schizophrenia Research, 62, 147-153. doi:10.1016/S0920-9964(02)00346-8

[20] Manoach, D.S., Thakkar, K.N., Stroynowski, E., et al. (2010) Reduced overnight consolidation of procedural learning in chronic medicated schizophrenia is related to specific sleep stages. Journal of Psychiatric Research, 44, 112-120. doi:10.1016/j.jpsychires.2009.06.011

[21] Ferrarelli, F., Peterson, M.J., Sarasso, S., et al. (2010) Thalamic dysfunction in schizophrenia suggested by whole-night deficits in slow and fast spindles. America Journal of Psychiatry, 167, 1339-1348.

[22] Ferrarelli, F. and Tononi, G. (2011) The thalamic reticular nucleus and schizophrenia. Schizophrenia Bulletin, 37, 306-315. doi:10.1093/schbul/sbq142

[23] Benson, K. and Zarcone, V.P. (1994) Schizophrenia. In: 
Kryger, M., Roth, T. and Dement, W., Eds., Principles and Practices of Sleep Medicine, Elsevier Limited, Philadelphia.

[24] Ancoli-Israel, S., Martin, J., Jones, D.W., et al. (1999) Sleep-disordered breathing and periodic limb movements in sleep in older patients with schizophrenia. Biological Psychiatry, 45, 1426-1432. doi:10.1016/S0006-3223(98)00166-8

[25] Waters, F., Naik, N. and Rock, D. (2012) Sleep-disordered breathing in psychiatric inpatients: An audit. Schizophrenia Research (under revision).

[26] Sharafkhaneh, A., Giray, N., Richardson, P., et al. (2005) Association of psychiatric disorders and sleep apnea in a large cohort. Sleep, 28, 1405-1411.

[27] Winkelman, J.W. (2001) Schizophrenia, obesity, and obstructive sleep apnea. Journal of Clinical Psychiatry, 62, 8-11. doi:10.4088/JCP.v62n0103

[28] Wirshing, D.A., Pierre, J.M. and Wirshing, W.C. (2002) Sleep apnea associated with antipsychotic-induced obesity. Journal of Clinical Psychiatry, 63, 369-370. doi:10.4088/JCP.v63n0415f

[29] Kang, S.G., Lee, H.J., Jung, S.W., et al. (2007) Characteristics and clinical correlates of restless legs syndrome in schizophrenia. Progress in Neuro-Psychopharmacology \& Biological Psychiatry, 31, 1078-1083. doi:10.1016/j.pnpbp.2007.03.011

[30] Cohrs, S., Guan, Z., Pohlmann, K., et al. (2004) Nocturnal urinary dopamine excretion is reduced in otherwise healthy subjects with periodic leg movements in sleep. Neuroscience Letters, 360, 161-164. doi:10.1016/j.neulet.2004.02.056

[31] Kraus, T., Schuld, A. and Pollmacher, T. (1999) Periodic leg movements in sleep and restless legs syndrome probably caused by olanzapine. Journal of Clinical Psychopharmacology, 19, 478-479. doi:10.1097/00004714-199910000-00017

[32] Winokur, A. and Kamath, J. (2008) The effect of typical and atypical antipsychotic drugs on sleep in schizophrenia patietns. In: Monti, J.M., Jacobs, B.L. and Nutt D.J., Eds., Serotonin and Sleep: Molecular, Functional and Clinical Aspects, Springer, Berlin, 587-610. doi:10.1007/978-3-7643-8561-3 24

[33] DeMartinis, N.A. and Winokur, A. (2007) Effects of psychiatric medications on sleep and sleep disorders. CNS \& Neurological Disorders Drug Targets, 6, 17-29. doi:10.2174/187152707779940835

[34] Cohrs, S. (2008) Sleep disturbances in patients with schizophrenia: Impact and effect of antipsychotics. CNS Drugs, 22, 939-962. doi:10.2165/00023210-200822110-00004

[35] Bromundt, V., Koster, M., Georgiev-Kill, A., et al. (2011) Sleep-wake cycles and cognitive functioning in schizophrenia. The British Journal of Psychiatry, 187, 269-276.

[36] Waters, F., Faulkner, D., Naik, N., et al. (2012) Effects of polypharmacy on sleep in psychiatric inpatients Schizophrenia Research, 139, 225-228. doi:10.1016/j.schres.2012.05.013

[37] Argyropoulos, S.V., Wilson, S.J., Nutt, D.J. (2008) The effects of antidepressant drugs and 5-HT1a agonists on human sleep. In: Monti, J.M., Jacobs, B.L.and Nutt, D.J. Eds., Serotonin and Sleep: Molecular, Functional and Clinical Aspects, Springer, Berlin, 569-585.

[38] Kripke, D.F., Garfinkel, L., Wingard, D.L., et al. (2002) Mortality associated with sleep duration and insomnia. Archives of General Psychiatry, 59, 131-136. doi:10.1001/archpsyc.59.2.131

[39] Hofstetter, J.R., Lysaker, P.H. and Mayeda, A.R. (2005) Quality of sleep in patients with schizophrenia is associated with quality of life and coping. BMC Psychiatry, 5, 13. doi:10.1186/1471-244X-5-13

[40] Pompili, M., Lester, D., Grispini, A., et al. (2009) Completed suicide in schizophrenia: evidence from a casecontrol study. Psychiatry Research, 167, 251-257. doi:10.1016/j.psychres.2008.03.018

[41] Zarcone, V.P. and Benson, K.L. (1997) BPRS symptom factors and sleep variables in schizophrenia. Psychiatry Research, 66, 111-120. doi:10.1016/S0165-1781(96)02857-0

[42] Poulin, J., Daoust, A., Forest, G., et al. (2003) Sleep architecture, phasic activities and their clinical correlates in drug-naive schizophrenic patients. Schizophrenia Research, 62, 147-153. doi:10.1016/S0920-9964(02)00346-8

[43] Neylan, T.C., van Kammen, D.P., Kelley, M.E., et al. (1992) Sleep in schizophrenic patients on and off haloperidol therapy. Clinically stable vs relapsed patients. Archives of General Psychiatry, 49, 643-649. doi:10.1001/archpsyc.1992.01820080051008

[44] Feinberg, I., Koresko, R.L. and Gottlieb, F. (1965) Further observations on electrophysiological sleep patterns in schizophrenia. Comprehensive Psychiatry, 58, 21-24.

[45] Ferrarelli, F. and Benca, R.M. (2010) Neurophysiology and neuroimaging of human sleep. Cambridge University Press, Cambridge.

[46] Keshavan, M.S., Montrose, D.M., Miewald, J.M., et al. (2011) Sleep correlates of cognition in early course psychotic disorders. Schizophrenia Research, 131, 231-234. doi:10.1016/j.schres.2011.05.027

[47] Zarcone, V.P. and Benson, K.L. (1997) BPRS symptom factors and sleep variables in schizophrenia. Psychiatry Research, 66, 111-120. doi:10.1016/S0165-1781(96)02857-0

[48] Waters, F., Sinclair, C., Rock, D., et al. (2011) Daily variations in sleep-wake patterns and severity of psychopathology: A pilot study in community-dwelling individuals with chronic schizophrenia. Psychiatry Research, 187, 304-306. doi:10.1016/j.psychres.2011.01.006

[49] Tandon, R., Shipley, J.E., Eiser, A.S., et al. (1989) Association between abnormal REM sleep and negative symptoms in schizophrenia. Psychiatry Research, 27, 359-361. doi:10.1016/0165-1781(89)90151-0

[50] Holt, D.J., Lebron-Milad, K., Milad, M.R., et al. (2009) Extinction memory is impaired in schizophrenia. Biological Psychiatry, 65, 455-463. doi:10.1016/j.biopsych.2008.09.017

[51] Manoach, D.S., Cain, M.S., Vangel, M.G., et al. (2004) A 
failure of sleep-dependent procedural learning in chronic, medicated schizophrenia. Biological Psychiatry, 56, 951956. doi:10.1016/j.biopsych.2004.09.012

[52] Rao, M.L., Gross G, Strebel B, et al. (1994) Circadian rhythm of tryptophan, serotonin, melatonin, and pituitary hormones in schizophrenia. Biological Psychiatry, 35, 151-163. doi:10.1016/0006-3223(94)91147-9

[53] Mansour, H.A., Wood, J., Logue, T., et al. (2006) Association study of eight circadian genes with bipolar I disorder, schizoaffective disorder and schizophrenia. Genes, Brain, and Behavior, 5, 150-157. doi:10.1111/j.1601-183X.2005.00147.X

[54] Monti, J.M. and Monti, D. (2005) Sleep disturbance in schizophrenia. International Review of Psychiatry, 17, 247-253. doi:10.1080/09540260500104516

[55] Kishi, T., Kitajima, T., Ikeda, M., et al. (2009) Association study of clock gene (CLOCK) and schizophrenia and mood disorders in the Japanese population. European Archives of Psychiatry and Clinical Neuroscience, 259, 293-297. doi:10.1007/s00406-009-0869-4

[56] Sarkar, S., Katshu, M.Z., Nizamie, S.H., et al. (2010) Slow wave sleep deficits as a trait marker in patients with schizophrenia. Schizophrenia Research, 124, 127-133. doi:10.1016/j.schres.2010.08.013

[57] Keshavan, M.S., Diwadkar, V.A., Montrose, D.M., et al. (2004) Premorbid characterization in schizophrenia. The
Pittsburgh High Risk Study World Psychiatry, 3, 163168.

[58] Buysse, D.J., Reynolds, C.F., Monk, T.H., et al. (1989) The pittsburgh sleep quality index: A new instrument for psychiatric practice and research. Psychiatry Research, 28, 193-213. doi:10.1016/0165-1781(89)90047-4

[59] Netzer, N.C., Stoohs, R.A., Netzer, C.M., et al. (1999) Using the Berlin questionnaire to identify patients at risk for the sleep apnea syndrome. Annals of Internal Medicine, 131, 485-491.

[60] Buysse, D.J., Monk, T.H., Carrier, J., et al. (2005) Circadian patterns of sleep, sleepiness, and performance in older and younger adults. Sleep, 28, 1365-1376.

[61] Johns, M.W. (1991) A new method for measuring daytime sleepiness: The Epworth sleepiness scale. Sleep, 14 540-545.

[62] Sugishita, K., Yamasue, H. and Kasai, K. (2010) Continuous positive airway pressure for obstructive sleep apnea improved negative symptoms in a patient with schizophrenia. Psychiatry and Clinical Neurosciences, 64, 665. doi:10.1111/j.1440-1819.2010.02146.x

[63] Boufidis, S., Kosmidis, M.H., Bozikas, V.P., et al. (2003) Treatment outcome of obstructive sleep apnea syndrome in a patient with schizophrenia: Case report. International Journal of Psychiatry in Medicine, 33, 305-310. 